University of Nebraska - Lincoln

DigitalCommons@University of Nebraska - Lincoln

$3-5-2021$

Evaluating person-centered factors associated with brain-computer interface access to a commercial augmentative and alternative communication paradigm

Kevin Pitt

Jonathan S. Brumberg

Follow this and additional works at: https://digitalcommons.unl.edu/specedfacpub

Part of the Special Education and Teaching Commons

This Article is brought to you for free and open access by the Department of Special Education and Communication Disorders at DigitalCommons@University of Nebraska - Lincoln. It has been accepted for inclusion in Special Education and Communication Disorders Faculty Publications by an authorized administrator of DigitalCommons@University of Nebraska - Lincoln. 


\title{
Evaluating person-centered factors associated with brain-computer interface access to a commercial augmentative and alternative communication paradigm
}

\author{
Kevin M. Pitt, $\mathrm{PhD}^{1}$ and Jonathan S. Brumberg, $\mathrm{PhD}^{2}$ \\ 1 Department of Special Education and Communication Disorders, \\ University of Nebraska-Lincoln, Lincoln, Nebraska, USA \\ 2 Department of Speech- Language-Hearing: Sciences \& Disorders, \\ University of Kansas, Lawrence, Kansas, USA
}

Correspondence - Kevin M. Pitt, PhD, Department of Special Education and Communication Disorders, University of Nebraska-Lincoln, 357 Barkley Memorial Center, PO Box 830738, Lincoln, NE 68583-0738; kevin.pitt@unl.edu

ORCID

Kevin M. Pitt http://orcid.org/0000-0003-3165-4093

Jonathan S. Brumberg http://orcid.org/0000-0001-5739-968X

\begin{abstract}
Current BCI-AAC systems largely utilize custom-made software and displays that may be unfamiliar to AAC stakeholders. Further, there is limited information available exploring the heterogenous profiles of individuals who may use BCI-AAC. Therefore, in this study, we aimed to evaluate how individuals with amyotrophic lateral sclerosis (ALS) learned to control a motor-based BCI switch in a row-column AAC scanning pattern, and person-centered factors associated with BCI-AAC performance. Four individuals with ALS completed 12 BCI-AAC training sessions, and three individuals without neurological impairment completed $3 \mathrm{BCI}-\mathrm{AAC}$ training sessions. To assess personcentered factors associated with BCI-AAC performance, participants completed both
\end{abstract}

Citation: Kevin M. Pitt \& Jonathan S. Brumberg (2021): Evaluating person-centered

factors associated with brain-computer interface access to a commercial

augmentative and alternative communication paradigm, Assistive Technology, DOI:

10.1080/10400435.2021.1872737

Copyright (C) 2021 RESNA; published by Taylor \& Francis. Used by permission.

Accepted 4 January 2021; published 5 March 2021. 
initial and recurring assessment measures including levels of cognition, motor ability, fatigue, and motivation. Three of four participants demonstrated either BCI-AAC performance in the range of neurotypical peers, or an improving BCI-AAC learning trajectory. However, BCI-AAC learning trajectories were variable. Assessment measures revealed that two participants presented with a suspicion for cognitive impairment yet achieved the highest levels of BCI-AAC accuracy with their increased levels of performance being possibly supported by largely unimpaired motor skills. Motor-based BCI switch access to a commercial AAC row-column scanning may be feasible for individuals with ALS and possibly supported by timely intervention.

Keywords: amyotrophic lateral sclerosis, augmentative and alternative communication, brain-computer interface, scanning, switch, translation

Noninvasive brain-computer interfaces (BCIs) commonly use electroencephalography (EEG) to provide means for accessing augmentative and alternative (AAC) communication devices using an individual's neurological activity alone without physical interaction (BCI-AAC; e.g., J. S. Brumberg et al., 2018). Such a connection through EEG circumvents the requirement for individuals to possess a reliable form of physical movement for accessing communication. Currently, there is no clinical pathway (assessment, selection, provision, and insurance funding) for clinical use of BCI-AAC devices, though some are beginning to become commercially available (e.g., the Intendix speller, g.tec medical engineering), highlighting the need for validated clinical practices involving BCI-AAC. However, while ongoing research focuses on improving BCI-AAC access to assistive technology devices (e.g., Gosmanova et al., 2017; Thompson et al., 2014; Zickler et al., 2011), BCI-AAC paradigms still largely utilize custom-made software and displays for eliciting targeted neurological control signals. The lack of consistency between custom-made BCI-AAC software and existing AAC devices and paradigms may impede the continuity of AAC intervention across the disease course, increasing an individual's emotional struggle and learning demands by requiring an individual to learn multiple forms of AAC (Blain-Moraes et al., 2012; Liberati et al., 2015), and possibly leading to device abandonment (Johnson et al., 2006). For instance, an individual may be required to learn to control and navigate a commercially available AAC system using eye-gaze or switch access before transitioning to BCI-AAC as motor impairment progress (Pitt, Brumberg, Pitt et al., 2019).

Scanning interfaces have a long history in traditional AAC implementation for providing AAC access to adults (e.g., Beukelman et al., 2007; 
Fried-Oken et al., 2015), and children (e.g., McCarthy et al., 2006) who find direct item selections via methods such as touch, or eye-gaze, ineffective or inefficient (Beukelman \& Mirenda, 2013). Broadly, during row-column scanning, communication items are presented by the communication device, or trained communication partner, in a set pattern (e.g., each row is highlighted and, following a selection, each column until the target item is reached). To select an item, the individual must wait until the communication partner or device scans to the desired item, and then perform a predetermined action for selection such as pressing a switch (Beukelman \& Mirenda, 2013). Due to clinician familiarity with switch access, and the established history of utilizing switches for scanning-based AAC access in clinical practice, BCI-based access to commercial AAC scanning paradigms is a logical, strong, and familiar framework for translating BCI-AAC technology into clinical scenarios. Motorbased BCI-AACs, in particular, are intuitively similar to physical switch access methods in which sensorimotor EEG activity related to physical, attempted, and imagined motor movements (i.e., motor imagery) can be decoded for device control. Specifically, motor-based BCI-AACs use event-related desynchronization (Pfurtscheller \& Da Silva, 1999), characterized by a decrease of power in the mu sensorimotor band and associated with cortical engagement in an imagined, attempted, or physical task, to activate the BCI-AAC switch (see Pitt et al., 2019, for a review of BCI-AAC methods). In this regard, Friedrich et al. (2009) investigated motor imagery-based BCI-AAC access for eight neurotypical participants, one individual with ALS who had limited upper mobility, and one individual with thoracic outlet syndrome without motor impairment. Participants completed a 10- session training protocol where they learned to select a target item from a set of four during automatic linear scanning via motor imagery (i.e., mental re-creation of a task without physical execution). While individual differences between the two participants with motor impairment and the neurotypical peers were not fully explored, overall results found that BCI-AAC performance was variable both within and across participants, with the participants' mean accuracy increasing from $35 \%(S D=14)$ in session one to its peak in Session $8(57 \%, S D=20$, chance accuracy $25 \%)$.

Building upon these foundations, motor imagery-based BCI-AAC access to larger AAC-style matrices was subsequently assessed by both Scherer et al. (2015) and J. S. Brumberg et al. (2016) during single 
training sessions. Scherer et al. (2015) evaluated single-session BCIAAC performance by 14 adults with cerebral palsy in a row-column scanning paradigm of a $3 \times 3$ display of graphical symbols (e.g., fruit). As motor imagery strategies can take time to learn, motor execution was used by one participant as an alternative, though brain signals were used for decoding and not any physical movement-related biomarkers. They found that while three participants were unable to successfully control the BCI-AAC, eleven participants achieved control above chance levels. Finally, J. S. Brumberg et al. (2016) evaluated a means to elicit sensorimotor rhythms using motor imagery-based access to a commercial Tobii- Dynavox page set incorporating a $4 \times 3$ matrix using motor imagery. Real-time feedback was not provided during this study and predicted online accuracy was calculated offline via a twofold cross validation. Six neurotypical individuals and one individual with ALS completed the BCI-AAC training session with neurotypical participants achieving a mean accuracy of 60\% (range: 55.763.55\%), and the individual with ALS achieving 62.6\% accuracy. Taken together, prior research provides strong support for continued evaluation of BCI-AAC approaches that allow for switch access to scanning paradigms. However, more research is needed to advance translation and place BCI-AAC in existing AAC frameworks and bolster clinician familiarity. Furthermore, the largely limited durations of these studies and discussed performance variability across sessions (Friedrich et al., 2009) did not permit examination of learning trajectories, and the aforementioned studies provide limited discussion regarding cognitive-sensory-motor factors that may impact BCI-AAC success. For instance, factors such as attention (Geronimo et al., 2016), motivation (Nijboer et al., 2010), fatigue (Myrden \& Chau, 2015), and energy levels (Geisler \& Polich, 1990) can impact BCI-AAC success. Therefore, taken together, further details regarding how individuals learn BCIAAC control, and how person-centered factors impact BCI-AAC outcomes will help elucidate clinically based assessment guidelines (see K. M. Pitt \& S. J. Brumberg, 2018a, for a review on factors that may influence BCI-AAC success).

Current advancements in motor-based BCI-AAC switches primarily focus on AAC control via motor imagery, the mental recreation of an action without physical execution, regardless of the individual's physical motor abilities. However, focus group findings from Liberati et al. 
(2015) reveal that individuals with ALS highly value AAC devices that can adapt to their changing sensory-cognitive-motor profile, exploiting the strongest current communication channel both in the short and long terms. This concept of ability-based AAC design seeks to develop AAC devices that support access across the lifespan/ disease course and emphasizes the role of creating AAC systems that can adapt to the individual's changing needs, instead of requiring the individual to adapt to the AAC technology (Light et al., 2019). The requirement for motor imagery only then shifts a burden to individuals that use BCIAAC who may have some amount of effortful or possibly unreliable voluntary motor ability and find motor imagery difficult to master. Furthermore, physical practice may facilitate improved first-person motor imagery performance or attempted movements (a strategy associated with improved motor imagery-based BCI-AAC success; Neuper et al., 2005) by supporting recall of the physical action from memory (Halder et al., 2011; Vuckovic \& Osuagwu, 2013). Thus, providing timely BCIAAC access via motor execution early in the disease course for those with progressive neuromotor disorders, before motor movements become severely impaired, may help provide a strong foundation for an individual's transition to a motor imagery/motor attempt strategy after progressive paralysis prevents efficient physical movements (Velliste et al., 2008). For instance, an individual may initially access a BCIAAC system via motor execution or multimodal AAC methods (e.g., $\mathrm{BCI}-\mathrm{AAC}$ and eye-gaze) early in the disease course, with the individual choosing their method of AAC access depending upon factors such as fatigue, motor ability, social, and environmental factors, then transition to BCI-AAC-only as overt behavior diminishes. Thus, additional research is needed to evaluate motor-based BCI-AAC switch access to commercial AAC devices by decoding brain activity associated with residual motor movements to pursue a goal of person-centered AAC continuity. Therefore, in the present study, we (a) evaluate the individual learning trajectories of four participants with ALS while mastering automatic row-column scan selection control via a motor-based BCI-AAC switch using physical and/or imagined movements based on individual preference; and (b) explore how an individual's initial cognitivesensory-motor profiles; and recurring factors (i.e., fatigue, motivation, and time since last meal) impact $\mathrm{BCI}-\mathrm{AAC}$ performance to help inform BCI-AAC assessment. 


\section{Methods}

\section{Participants}

Four individuals with a diagnosis of ALS (participants A1-A4, ages 3864, mean 52 years, 2 females, all right-handed; see Table 1 in Supplementary Materials), and three neurotypical individuals (T1-T3, ages 23-60, mean 41 years, 2 females, all right-handed) completed the study. Participants did not report or demonstrate any vision or hearing loss that may impair BCI-AAC use. The study was approved by the University of Kansas institutional review board with all participants providing informed consent. All participants were financially compensated.

\section{EEG and BCI-AAC Recording and data processing}

EEG recordings were collected at a sampling rate of $256 \mathrm{~Hz}$ via 62 active electrodes (g.HIAmp, g.tec) arranged according to the 10-10 standard (Oostenveld \& Praamstra, 2001), with forehead ground electrode, reference to the left earlobe, and a notch filter from 58 to $62 \mathrm{~Hz}$ to eliminate powerline artifacts.

\section{BCI-AAC calibration}

Prior to online BCI-AAC control, and similar to calibration procedures for eye-gaze AAC access, BCI-AAC calibration data were collected from 90 trials that included 60 trials of motor execution during which participants were instructed to move, or attempt to move, either their upper or lower limbs (e.g., 30 trials of left hand or foot and 30 trials of right hand or foot execution), and 30 trials of rest. The calibration data were then processed offline using custom routines in MATLAB (The MathWorks, Natick, MA) for setting online BCI-AAC control parameters. Processing included standard preprocessing (e.g., sensorimotor $(8-25 \mathrm{~Hz}$ ) bandpass filtering, windowing $0-2 \mathrm{~s}$ aligned to the instruction onset), common spatial filtering of the bandpass filtered EEG signal (e.g., first and last 3 common spatial filters selected), and decoder estimation (e.g., discriminant weight estimation and regularization parameter optimization). The specific frequency ranges used were tailored to each participant, some were based on a general broadband sensorimotor range 
(e.g., 8-25 Hz), while others were narrowband ranges (e.g., mu: approx 8-15 Hz and beta: 15-25 Hz). A leave-one-out cross-validation procedure was used to simultaneously optimize common spatial filter regularization (e.g., fine to coarse spatial filters) and discriminant weights for the regularized common spatial pattern decoding algorithm with linear discriminant analysis (Lotte \& Guan, 2010). Optimal parameter values and frequency ranges used were chosen by maximizing the area under the curve (AUC). While common spatial filters were data driven, those chosen typically had filter values that reflected sensorimotor processing (e.g., left and right C/CP electrodes). All weights were stored for real-time decoding.

\section{BCI-AAC control}

Participants completed copy spelling tasks during online BCI-AAC control by making letter selections from a $7 \times 5$ keyboard display including letters A-Z, space, and backspace, similar to those available on commercial AAC devices (see Figure 1) in an automatic row-column scanning pattern. Similar to existing switch-scanning AAC interfaces, the keyboard automatically advanced a blue selection box in a linear fashion through all possible rows in the graphical display, while the individuals remained at rest. Switch activation and subsequent row selection occurred when the BCI-AAC detected sensorimotor brain activity related to an executed movement (J. Brumberg et al., 2018). Following row selection, the AAC device automatically advanced the selection box in a linear fashion through all possible columns within the row, with final letter selection upon a second BCI-AAC switch activation. A scanning rate of $2 \mathrm{~s}$ per item with an interstimulus duration of $1 \mathrm{~s}$ was employed. To indicate a selection was made, the blue box briefly turned dark gray, and the selected item appeared in the selection window located at the top of the display (see Figure 1).

Sensorimotor activity utilized for BCI-AAC control was modulated by motor execution or imagery of the upper (for participants A1-A3), or lower limbs (for A4). Drawing from existing practices in AAC assessment, selection of the limb used for BCI-AAC control was based upon participant preference, physical motor skills, and calibration results (i.e., predicted BCI-AAC accuracy). For instance, while unused at this time, A4 owned two knee switches for use with his recently purchased AAC 


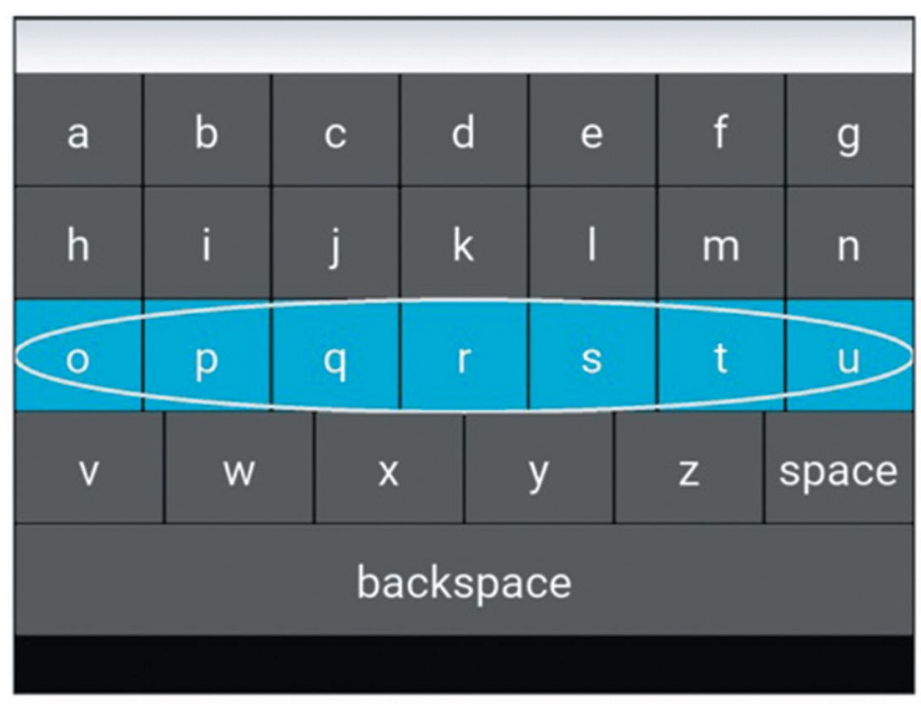

Figure 1. Motor (imagery) BCI-AAC Interface. The motor (imagery) BCI-AAC interface, depicting the third row highlighted, the feedback circle in white, and the empty selection window at the top of the image.

device. Therefore, in addition to having less motor impairment in his lower versus upper limbs, he wished to be consistent between use of the BCI-AAC and his physical switches to potentially increase functionality should the BCI-AAC system become a viable option for AAC access in the future. To accurately select a target item in our study, the BCI-AAC must accurately decode both "select" trial decisions, during which the individual performs motor execution and elicits a change in the sensorimotor rhythm, in addition to "do not select/continue scanning" trial decisions, where the individual remains relaxed. Visual feedback was provided to participants regarding how close the BCI-AAC was to making a selection in order to help support motor learning during online control. This feedback was provided in the form of a circle overlaid on the current item that decreased in size as the BCI-AAC algorithm became more confident in predicting a "select" command and increased size as confidence decreased (see Figure 1).

\section{Online BCI-AAC training sessions}

Participants with a diagnosis of ALS completed a total of 12 online BCIAAC training sessions. For comparison of initial BCI-AAC performance, individuals without neurological impairment completed three BCI-AAC 
trainings. During each session, participants completed approximately 300 select/no-select trials for copy spelling per session. Spelling time was limited to approximately $20 \mathrm{~min}$. Participants began training sessions with a set of words with four to five letters evenly distributed across the keyboard (approximately 20-30 selections per word are needed for perfect spelling), though we also allowed participants to choose words on their own to increase buy-in and motivation (e.g., participant and study team member names, day of the week). For participants $\mathrm{A} 1$ and $\mathrm{A} 2$, training sessions were completed in the laboratory setting (i.e., an electrically shielded booth, with the door open to allow for communication with the participant throughout the BCI-AAC session). However, due to travel challenges, BCI-AAC sessions for A3 and A4 were in a quiet room in their home setting.

\section{BCI-AAC learning trajectories}

BCI-AAC accuracy was determined using Cohen's kappa, which represents the overall agreement between the BCI-AAC-decoded output, and targets for both select and "keep scanning" trials (Note: The linear nature of automatic row-column scanning leaves only one way to select a letter). Cohen's kappa values have been previously used to assess BCI-AAC outcomes (e.g., Daly et al., 2013; Zhang et al., 2019) and can be considered as an accuracy measure weighted by both true response and false errors, with values of 0 to 0.20 indicating no to slight agreement between the BCI-AAC output and user intention, 0.21 to 0.4 as fair agreement, 0.41 to 0.6 as moderate agreement, 0.61 to 0.8 as substantial agreement and .81 to 1 as almost perfect agreement (e.g., McHugh, 2012). Negative kappa values are possible and indicate performance below chance levels. For the row-column scanning paradigm, Cohen's kappa is suitable for outcome assessment as it adjusts for bias in trial/condition numbers, representing relative increases and decreases in true negatives (correct non-selects) and positives (correct selects), as well as false negatives (the BCI-AAC incorrectly does not select an item) and false positives (the BCI-AAC incorrectly selects an item). This consideration is important since multiple no-selection/continue scanning trials are required to scan through the nontarget letters in row-column scanning and complete a target selection task. For auto-scanning paradigms, traditional measures of percent accuracy 
(number of correct selects and non-selects/total trials) are weighted toward performance of non-selection tasks, skewing this performance metric. Furthermore, Cohens Kappa was chosen for this study as the metric reflects all accurate selections made by the participant, and not only correct letter selections. Utilizing a metric that will incorporate all accurate selections is important for establishing feasibility and generalization of the proposed row-column paradigm to other switch techniques (e.g., automatic linear scanning). In the row-column scanning paradigm utilized in this study, an individual must make two switch selections (row and column) to select the appropriate letter. In comparison, linear automatic scanning only requires the individual to activate the switch on one occasion. The row-column scanning paradigm was chosen for this study as it may help increase communication rate (Beukelman \& Mirenda, 2013). However, while metrics such as number of letters selected are an important measure for future clinical BCI-AAC performance, these metrics limit the assessment of switch performance to only displays that require two switch activations for letter selection and does not allow for a full representation of switch performance. By choosing accuracy defined by selection rates the performance on our BCI-AAC may be generalized to other selection paradigms where any number of selections are needed for final communicative item choice. For instance, more selections are required to spell a word via an alphanumeric keyboard than a single icon representing a full word. Therefore, number of letters selected is representative of improved skill learning in the area of making item selections. Thus, performance metrics such as Cohen's kappa that account for changes in both true positive and negative performance are ideal for reflecting skill learning in this BCI-AAC paradigm that generalizes to other paradigms that use item selections.

To characterize the performance trajectory across the 12 training sessions we assessed participants' mean kappa value, kappa range, 95\% confidence interval, overall performance increase and learning slope. Based upon prior research (e.g., Ahn \& Jun, 2015), it was expected that BCI-AAC performance would be variable both within and between participants. BCI-AAC performance variability means the final study session may not necessarily be representative of an individual's overall performance improvement. Therefore, performance increase was calculated as highest performance minus lowest performance to best characterize 
the individual's potential to utilize the BCI-AAC system and is interpreted alongside other descriptive measures to describing BCI-AAC performance.

\section{Assessment}

\section{Initial cognitive-sensory-motor assessment}

Before starting online BCI-AAC training, participants with ALS also completed a range of assessments to help elucidate their unique profile. Assessments were chosen to capture a variety of cognitive, sensory and motor aspects, and were chosen based on current literature regarding BCI-AAC assessment and clinical assessment for those with neuromotor disorder. All assessment protocols were administered in a quiet room without distraction. Assessment protocols included (a) ALS Cognitive Behavioral Screen (ALS-CBS; Woolley et al., 2010), screening the areas of attention, concentration, tracking and monitoring, and initiation and retrieval; (b) the cognitive and motor portions of the BCI-AAC screener from K. Pitt and Brumberg (2018b), with the cognitive section including the areas of comprehension and orientation, following directions, attention and working memory, cognitive motor learning/ abstract problem-solving, and the motor section focusing on oculomotor movement, upper and lower limb movement, and positioning. We decided to use both the ALS-CBS and BCI-AAC screener in participant assessment because, as the screener focuses on BCI-AAC relevant tasks, cognitive scores were shown to differ between the two protocols (K. M. Pitt \& J. S. Brumberg, 2018b). These score differences suggest the two screening protocols are assessing different skills. Further, participants completed (a) the ALS-Functional Rating Scale (ALS-FRS; Cedarbaum \& Stambler, 1997) assessing motor function in the bulbar (speech, salivation, swallowing), fine motor (handwriting, cutting food, dressing and hygiene), gross motor (walking, climbing stairs, turning in bed and adjusting bed clothes), and breathing domains (Bacci et al., 2016); (b) the Bimanual Fine Motor Function (BFMF; Beckung \& Hagberg, 2002); and (c) Manual Ability Classification System (MACS; Eliasson et al., 2006). Both the BFMF and the MACS provide complementary assessment regarding activity limitation due to motor impairment in the upper limbs (Elvrum et al., 2016). Due to the limited number of data points $(n=4)$ for initial screening measures for those with ALS, participant results will be descriptively related to BCI-AAC performance. 
Recurring measures

Participants rated their current level of fatigue ( 1 being normal, to 9 extremely fatigued) and motivation ( 1 being extremely unmotivated to 9, extremely motivated to use the BCI-AAC system) on a 9-point number scale, along with indicating the time elapsed since their last meal on a recurring basis prior to each session since these factors may change over time (e.g., Thompson, 2018). Within-subject correlations across sessions for these recurring measures were assessed using a within-subject Spearman's rank order correlation using the 12 data points collected at the beginning of each online BCI-AAC training session.

\section{Results}

\section{Initial cognitive assessments}

For the ALS-CBS, the maximum score is 20 , with a total score of $<17$ indicating a concern for cognitive impairment. Scores differed among participants, with scores of 14, 15, 19, and 19 for participants A4, A1, A2, and A3, respectively. These ALS-CBS scores indicate a concern of cognitive impairment for both participants $\mathrm{A} 1$ and $\mathrm{A} 4$. The cognitive portion of the BCI-AAC screener has a maximum score of 24, and participants A1, A2, A3, and A4 scored 22, 22, 24, and 24, respectively, indicating the presence of BCI-AAC-related cognitive skills for all participants. Results are provided in Table 2 within Supplementary Materials

\section{Initial functional motor control and manual ability assessments}

Motor assessment guided by the BCI-AAC screener revealed participant A1 was ambulatory with no impairments noted in the upper or lower limbs. For the upper limbs, participant A2 had limited range of motion, weakness, and decreased ability to grasp/ grip, with increased right-side impairment. For the lower limbs, participant A2 was ambulatory with a walker, with decreased range of motion and increased leg spasticity when standing. Participant A3 had a severe upper limb impairment with physical abilities limited to movement of her index finger on left hand, and limited ability to grip with the right hand. For the lower limbs, participant A3 was not ambulatory, retaining the ability to lift her thighs and 
move her toes. For the upper limbs, participant A4 had limited range of motion, weakness, and decreased ability to grasp/ grip bilaterally. Fine motor impairments were decreased for his left side. For the lower limbs, participant A4 reported minimal impairments and was ambulatory without assistance. No participants had difficulties with oculomotor control. The ALS-FRS has a maximum score of 40 , which may be broken down into the subdomains of bulbar (maximum score of 12), fine motor (maximum score of 12), gross motor (maximum score of 12), and breathing (maximum score of 4) (Bacci et al., 2016). Lower ALS-FRS scores indicate greater motor impairment. Scores varied by severity with A3 having the lowest total score of 15 (bulbar $=9$, fine motor $=0$, gross motor $=3$, breathing $=3$ ) and A1 having the highest total score of 33 (bulbar $=$ 9 , fine motor $=12$, gross motor $=9$, breathing $=3$ ). A2 had a total score of 30 (bulbar $=10$, fine motor $=8$, gross motor $=8$, breathing $=4$ ), and A4 had a total score of 26 (bulbar $=9$, fine motor $=4$, gross motor $=10$, breathing = 3). For the MACS and BFMF, scores range from 1 to 5 but, in contrast to the ALS-FRS, higher scores indicate greater motor impairments. Scores for the MACs included 1, 2, 2, and 5 for participants A1, $\mathrm{A} 2, \mathrm{~A} 4$, and A3, respectively, and 1, 2, 3, and 5 on the BFMF for participants A1, A2, A4, and A3, respectively. Results are provided in Table 3 within Supplementary Materials.

\section{BCI-AAC learning trajectories}

\section{Neurotypical learning trajectories}

Neurotypical participant 1. For neurotypical participant T1, her average BCI-AAC accuracy across three sessions was 0.412 (range: $0.383-$ 0.447; $S D=.032$ ), reaching levels of moderate agreement. Her overall increase in BCI-AAC accuracy (highest performance minus lowest performance) was 0.064. Cohen's kappa values and 95\% confidence intervals for each session are provided in Figure 2 and Supplementary Materials Table 4 .

Neurotypical participant 2. For neurotypical participant T2, his average BCI-AAC accuracy across three sessions was 0.689 (range: 0.657$0.734 ; S D=.040$ ), reaching substantial agreement levels, though the $95 \%$ confidence interval ranges extend into the range of substantial to almost perfect agreement for Session 3. His increase in BCI-AAC accuracy was 0.099. Cohen's kappa values and 95\% confidence intervals for each session are provided in Figure 2 and Supplementary Materials Table 4. 
Neurotypical participant 3. For neurotypical participant T3, her average BCI-AAC accuracy across three sessions was 0.387 (range: 0.062$0.568 ; S D=.283$ ), reaching the upper levels of moderate agreement, though the $95 \%$ confidence interval range extends into the range of substantial agreement for Sessions 2 and 3. Her overall increase in BCI-AAC accuracy was 0.506 , showing a large increase in BCI-AAC learning between Sessions 1 and 2. Cohen's kappa values and 95\% confidence intervals for each session are provided in Figure 2 and Supplementary Materials Table 4.

\section{Learning trajectories of participants with ALS}

Participant A1. For participant A1, her average BCI-AAC accuracy across all 12 sessions was 0.333 (range: $0.020-0.544 ; S D=.151$ ) averaging fair levels of agreement, and ranging from no to mdoerate agreement, though the 95\% confidence interval range extends into the range of substantial agreement for Sessions 6 and 7. She had an overall increase of 0.524 in BCI-AAC accuracy. Cohen's kappa values and 95\% confidence intervals for each session are provided in Figure 2 and Supplementary Materials Table 5, which shows a learning trajectory slope of 0.0023 (kappa as a function of session) and a large increase in BCI-AAC learning between Sessions 1 and 2 (0.425).

Participant A2. For participant A2, his average BCI-AAC accuracy across all 12 sessions was 0.139 (range: -.051- 0.340; $S D=.117$ ) averaging no to slight agreement, and ranging from less than chance to fair agreement levels. Whereas overall his BCI-AAC performance was highly variable, the $95 \%$ confidence interval range extends into the range of moderate agreement for Sessions 1 and 4, and an overall increase of 0.340 in BCI-AAC. Cohen's kappa values and 95\% confidence intervals for each session are provided in Figure 2 and Supplementary Materials Table 6. The learning trajectory for participant A2 was calculated with a slope of 0.0033 .

Participant A3. For participant A3, her average BCI-AAC accuracy across all 12 sessions was -0.01 (range: -.017-0.13; $S D=.096$ ) indicating below chance levels, and ranging from below chance levels to no-slight agreement. Her overall increase in BCI-AAC accuracy was 0.147. Cohen's kappa values and 95\% confidence intervals for each session are provided in Figure 2 and Supplementary Materials Table 7. Although variable, visual inspection of the data in Figure 2 demonstrates 

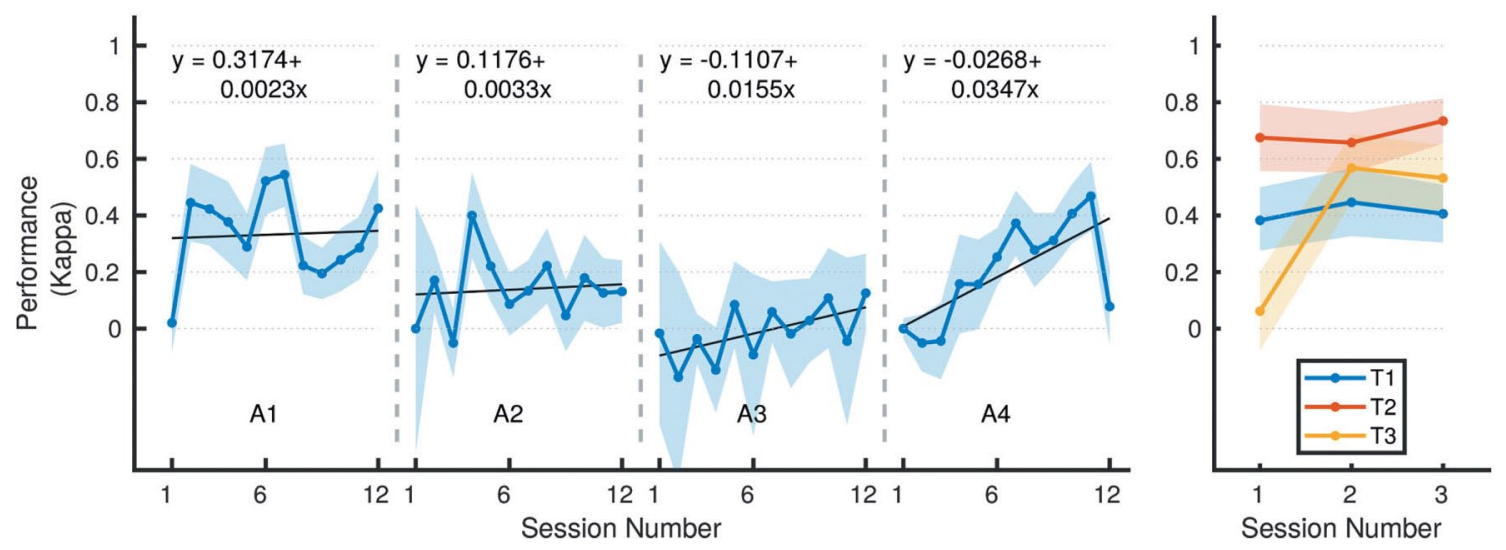

Figure 2. BCI-AAC learning trajectory for all participants. Left: Cohen's Kappa values denoting the BCI-AAC learning trajectories for participants with ALS (participants A1, $\mathrm{A} 2, \mathrm{~A} 3$, and $\mathrm{A} 4$ ) across the $12 \mathrm{BCI}-\mathrm{AAC}$ training sessions. A linear regression fit to the trajectories is shown in black and the line equation given for all participants. Ninetyfive percent confidence intervals are shown in the shaded areas for each participant. Right: Cohen's Kappa values denoting the BCI learning trajectories for neurotypical control participants T1, T2, and T3 across three BCI-AAC training sessions. Ninetyfive percent confidence intervals are shown in the shaded areas for each participant.

her learning trajectory began at Session 3 and is reflected by a slope of 0.0155. However, the correlation between session number and BCI-AAC performance approached but did not reach significance $\left(r_{s}(10)=.517\right.$, $p=.085)$.

Participant A4. For participant A4, his average BCI-AAC accuracy across all 12 sessions was .199 (range: -0.05-0.47; $S D=.177$ ) indicating, on average, slight agreement, and ranging from below chance levels to moderate agreement. However, kappa values and confidence intervals increased into the upper levels of moderate agreement for Sessions 10 and 11 and an overall increase in BCI-AAC accuracy of 0.52. Cohen's kappa values and 95\% confidence intervals for each session are provided in Figure 2 and Supplementary Materials Table 8. For participant A4, session number is significantly and positively correlated to BCI-AAC performance $\left(r_{s}(10)=.699, p<.05\right)$. Based upon visual inspection of the graphs, an upward learning trajectory began at Sessions 3 to 4 and is reflected by a slope of 0.0347 , which was the largest slope for all participants with ALS. 


\section{Recurring measures}

Participant A1. Results indicate: (a) motivation ratings on a scale from 1 (extremely unmotivated) to 9 (extremely motivated) did not vary for participant 1 with a score of 9 (extremely motivated) prior to beginning each session; (b) fatigue ratings on a scale of 1 (normal) to 9 (extremely fatigued) taken prior to the start of each training session had an average rating of $3.75(S D=1.66)$, ranging from 1 (normal) to 6 (moderatehigh), and a nonsignificant correlation $\left(r_{s}(10)=-0.389, p=.212\right)$ with BCI-AAC accuracy (Cohen's kappa; range: 0.020-0.544; $M=0.333 ; S D$ $=.151$ ); and (c) time since last meal averaged 0.958 hours (range 0-3; $S D=1.25)$ with a nonsignificant correlation $\left(r_{s}(10)=0.000, p=1\right)$ with BCI-AAC accuracy.

Participant A2. Results indicate: (a) motivation had an average rating of 7.33 ( $S D=.888$ ), ranging from 6 (moderate-high) to 9 (extremely motivated) and a nonsignificant correlation $\left(r_{s}(10)=-0.000, p=1\right)$ with BCI-AAC accuracy (range: $-0.051-0.340 ; M=0.139 ; S D=0.117$ ); (b) fatigue had an average rating of $2.67(S D=1.56)$, from 1 (normal) to 5 (moderate), and a nonsignificant correlation $\left(r_{s}(10)=-0.495, p=.102\right.$ ) with BCI-AAC accuracy; and (c) time since last meal averaged 9.72 hours (range: $0.41-16 ; S D=6.77$ ), and a nonsignificant correlation $\left(r_{s}(10)=\right.$ $-0.331, p=.293$ ) with BCI-AAC accuracy. However, motivation ratings demonstrated a significant negative correlation $\left(r_{s}(10)=-.763, p<.05\right)$ with session number.

Participant A3. Results indicate: (a) motivation had an average rating of 8.58 ( $S D=.515)$, from 8 (fairly-extremely motivated) to 9 (extremely motivated) and a nonsignificant correlation $\left(r_{s}(10)=-0.416\right.$, $p=.178$ ) with BCI-AAC accuracy (range: $-0.17-0.13 ; M=-.01 ; S D=$ $0.096)$; (b) fatigue had an average rating of 3.88 ( $S D=1.63)$, from 1 (normal) to 7.5 (high), and a nonsignificant correlation $\left(r_{s}(10)=0.288\right.$, $p=.365$ ) with BCI-AAC accuracy; and (c) time since last meal averaged 2.16 hours (range $0.33-3 ; S D=1.06$ ), with a nonsignificant correlation $\left(r_{s}(10)=0.287, p=.365\right)$ with BCI-AAC accuracy. However, motivation ratings demonstrated a significant negative correlation $\left(r_{s}(10)=-0.857\right.$, $p=<.001$ ) with session number.

Participant A4. Results indicate: (a) motivation had an average rating of 7.42 ( $S D=.792)$, from 6 (moderate-high) to 8 (fairly- extremely motivated), and a nonsignificant correlation $\left(r_{s}(10)=-0.158, p=.625\right)$ with BCI-AAC accuracy (range: $-0.05-0.47 ; M=.199 ; S D=0.177$ ); (b) fatigue 
had an average rating of $5.49(S D=1.38)$, from 2 (normal-mild) to 7 (high), and a nonsignificant correlation $\left(r_{s}(10)=0.392, p=.208\right)$ with BCI-AAC accuracy; and (c) time since last meal averaged 1.8 hours (range 1-3.5; $S D=.634)$, with a nonsignificant correlation $\left(r_{s}(10)=0.044, p=\right.$ .892) with BCI-AAC accuracy. Motivation ratings demonstrated a nonsignificant correlation $\left(r_{s}(10)=0.118, p=.714\right)$ with session number.

\section{Discussion and conclusion}

\section{Feasibility for BCI-AAC access to clinical row-column scanning paradigms}

Overall, findings from this project provide support for translating motorbased BCI switch access to clinical, commercial AAC row-column scanning paradigms for individuals with ALS. This finding builds upon the work of J. S. Brumberg et al. (2016), Scherer et al. (2015), and Friedrich et al. (2009), for bridging the gap between BCI-AAC research practices and existing approaches to AAC access. Learning trajectories for the BCI$\mathrm{AAC}$ device in the present study were variable both between and within participants for those with ALS. However, only participant A2 was unable to demonstrate either a BCI-AAC performance in the range of neurotypical peers, or an improving BCI-AAC learning trajectory. In contrast, for more than one session, both A1 and A4 achieved levels of BCI-AAC control within the range of neurotypical controls, which was $0.38-0.73$ (we discounted the initial session for T3 as an outlier due to initial BCIAAC learning). Specifically, A1 achieved kappa performance of $0.46,0.42$, $0.52,0.54$, and 0.43 for Sessions 2, 3, 6, 7, and 12, respectively, and A4 achieved kappa performance of 0.41 and 0.47 for Sessions 10 and 11, respectively. Furthermore, A3 and A4 were able to demonstrate an improving BCI-AAC learning trajectory, and while the average BCI-AAC performance was low for A3 across sessions $(M=-0.01, S D=.096)$, these findings highlight that an individual with severe physical impairments may demonstrate BCI-AAC learning. However, extensive training may be needed for proficiency. Further, it is important to note that, while A3 could not spell a 4- to 5-letter word without multiple spelling errors rendering the word largely unreadable, individualized adaptions to the BCI$\mathrm{AAC}$ system were not performed during training to allow for comparison 
across participants. For instance, due to disease severity, A3 had multiple muscle spasms throughout BCI-AAC calibration and training, which likely decreased performance. Therefore, adaptions to improve signal processing such as online removal of muscle artifacts and combining signals from other modalities may increase overall performance values. Interpreting these findings in a feature-matching framework, A3 already uses an eye-gaze AAC system to support communication, environmental control, and internet browsing. Therefore, a visual, sensory BCI-AAC system such as the visual P300-speller may provide A3 with a more familiar AAC access method along her existing strengths, and lessen the burden associated with BCI-AAC learning.

\section{Cognitive and motor skills in relation to BCI-AAC performance}

\section{Motor skills}

While functional motor abilities have not been shown to relate to motor (imagery)-based BCI-AAC control (Geronimo et al., 2016; Kasahara et al., 2012), one's average amount of daily hand-arm movement can improve motor (imagery)-based BCI-AAC performance (Randolph et al., 2010; Rimbert et al., 2019). In line with the findings of Randolph et al. (2010) and Rimbert et al. (2019), the highest levels of performance for our study were achieved by participants A1 and A4 who both demonstrated the least level of motor impairment in the limb utilized for BCI-AAC control (i.e., A1 upper limb, and A4 lower limb). Relevant differences in motor ability between participants are especially apparent in the qualitative descriptions obtained by the BCI-AAC screener by indicating no impairment of the upper limb for A1, and A4 reporting his legs were "still strong," being ambulatory without assistance. In comparison to the BCI-AAC screener, other measures utilized for assessment of motor function were limited in elucidating differences between participants that were relevant to BCI-AAC control. Specifically, while the MACS and BFMF were able to quantify differences in upper limb ability, they do not assess function of the lower limb, which may be utilized for BCI-AAC control. Furthermore, the ALS-FRS assesses a range of functional motor abilities, including those related to upper, lower, and bulbar function. Therefore, while total ALS-FRS scores highlight that A1 had the least level of motor impairment and A3 had the highest level of motor impairment, overall, specific differences in 
motor abilities between participants are less clear. When applying bulbar, fine motor, gross motor and respiratory domain analysis to ALSFRS scores (Bacci et al., 2016), further differences were highlighted between participants. Domain results reveal that differences in total scores for A2 and A4 were primarily influenced by A2's negative perception of his fine motor abilities. Fine motor skills may play a role in BCI-AAC performance (Hammer et al., 2012). However, while score differences in the gross motor domain were minimal (i.e., $\mathrm{A} 1=9, \mathrm{~A} 4=10$, $\mathrm{A} 2=8, \mathrm{~A} 3=3$ ), they paralleled BCI-AAC performance best with A1 and A4 providing higher ratings (indicating less impairment) than A2 and A3. Nonetheless, domain scores do not clearly differentiate upper and lower limb functions or explore specific tasks that may be specifically utilized for BCI-AAC control. Therefore, taken together, these findings highlight the role of individualized assessment of motor function, as guided by tools such as the BCI-AAC screener (K. M. Pitt \& J. S. Brumberg, 2018b), alongside existing measures of motor function.

\section{Cognitive skills}

ALS-CBS scores reveal that both A1 and A4 presented with a suspicion for cognitive impairment, as characterized by ALS-CBS scores below 17 (Woolley, 2014). However, participants A1 and A4 achieved the highest levels of BCI-AAC performance. The finding of greater BCI-AAC performance for participants with ALS who have suspicion for cognitive deficits contrasts with previous findings for motor-imagery BCI-AACs, which found diminished performance for those with lower ALS-CBS scores (Geronimo et al., 2016). However, participants with suspected cognitive impairment in our study also presented with the least physical impairment in the limb used for BCI-AAC control. It is plausible providing an individual with a motor-based BCI-AAC device before loss of physical movement may lower the cognitive demands associated with the abstract nature of motor imagery performance. Therefore, starting BCI-AAC intervention early, before loss of motor function, may allow time for an individual to begin establishing BCI-AAC mastery. Hopefully, these early skills will support BCI-AAC control through motor imagery strategies later in the disease course, and despite possible cognitive decline. However, more research is needed to determine the benefits of early intervention on BCI-AAC performance. 


\section{BCI-AAC learning}

Each participant with ALS demonstrated their own unique BCI-AAC performance trajectory across the 12 training sessions. Based upon visualization of participant data, all neurotypical participants and three of four participants with ALS were able to demonstrate growth in around five 20-min sessions of approximately 300 trials (with 20-30 selections). Therefore, along with consideration of individual preferences, requiring at least five sessions to make informed decisions about motor-based BCI-AAC performance helps to establish research-based performance guidelines to inform clinical decision-making on BCI-AAC device selection. Regarding individual learning trajectories, the following characteristics were associated with promising outcomes observed within the first five BCI-AAC training sessions:

(1) BCI-AAC performance similar to that of neurotypical peers for at least two consecutive sessions (as with A1).

(2) beginning and maintaining an improving BCI-AAC performance trajectory within the first five sessions (as with A3 and A4). However, an extended number of sessions may be necessary for individuals showing variable/low BCI-AAC performance (such as A3), allowing time for further individualization of BCI-AAC parameters to improve performance stability and overall performance magnitude.

It is important to note these are just general guidelines based on a limited sample size, and the length of time an individual is provided for BCI-AAC learning should take into account factors beyond basic accuracy (e.g., stakeholder preferences, motivation). This means an extended number of sessions will likely be necessary. Furthermore, it is unclear whether these results generalize to other sensorimotor BCI-AAC paradigms, which may have differing cognitive-sensory-motor demands (e.g., Nijboer et al., 2010).

\section{Recurring measures in relationship to BCI-AAC performance}

Increasing levels of fatigue may negatively impact BCI-AAC performance (Talukdar et al., 2019). However, for our study, no significant correlations were identified between an individual's fatigue at the start of the session and subsequent performance. The lack of correlation between fatigue and BCI-AAC performance may be influenced by limited ratings of fatigue 
(average ratings of fatigue were 3.75, 2.67, 3.88 and 5.49 for A1-A4, respectively), and high levels of motivation. When considering motivation, on average, ratings were high for all participants with ALS. Participants reported a range of factors influencing their high levels of motivation, including the hope of using BCI-AAC as a communication method, interest in BCI-AAC technology, and a desire to help science. However, motivation ratings significantly decreased across sessions for A2 and A3. This decrease in motivation ratings may be due to low levels of BCI-AAC control. While pre-session ratings of motivation were not significantly correlated to performance in this study, possibly due to our participants' high levels of baseline motivation, previous findings have identified motivation as an important aspect in achieving BCI-AAC control (e.g., Kleih \& Kübler, 2015). Consideration of how to increase motivation during the early stages of motor learning may help support BCI-AAC success. For instance, whereas BCI-AAC studies have traditionally focused on copy spelling tasks, A4 reported his high levels of motivation were generally driven by his desire to attempt free spelling tasks and move to the next level of functional independence in BCI-AAC control. Free spelling tasks were planned for completion during this investigation once a criterion level of BCI-AAC accuracy was achieved through copy spelling training, namely, substantial levels of kappa agreement for two consecutive sessions. However, no participants with ALS achieved BCI-AAC performance of this level. Therefore, the incorporation of functional communication tasks beyond copy spelling may support sustained levels of BCI-AAC motivation helping improve outcomes across sessions. Finally, further investigation on the effects of medications and food intake on BCI-AAC performance over time may help elucidate why participant A2 was unable to establish higher levels of BCI-AAC control or a positive learning trajectory. For instance, similar to P300-BCI-AAC control, it is feasible that pharmaceutical effects (e.g., K. M. Pitt \& J. S. Brumberg, 2018a; Meador, 1998) and decreased food consumption (Geisler \& Polich, 1990) may have negatively impacted cognitive and BCI-AAC performance.

\section{Limitations}

The limited sample size of this study means further work is needed to confirm our findings, develop clinical guidelines for BCI-AAC devices based on motor execution and motor imagery, and expand results to 
inform assessment of other BCI-AAC techniques. While our limited sample size of individuals with ALS was sufficient to lay clinical groundwork, additional work is needed to generalize findings to other populations of individuals who may use BCI-AAC as an access method (e.g., brain stem stroke, upper spinal cord injury, locked-in syndrome, cerebral palsy) and for relating selection accuracy used in this study with communication success in alternative paradigms (e.g., letter spelling vs word, phrase, or symbol selection). Finally, it is important to note that to facilitate comparison across participants, apart from allowing A4 to use his lower limb for BCI-AAC control due to increased strength, the BCI-AAC system was not individualized or adapted to meet individual needs. Therefore, future research evaluating signal processing techniques, feedback methods, and training paradigms (e.g., feedback, intensity) may help support overall increase in BCI-AAC performance.

\section{Conclusion}

Considering BCI-AAC access to commercial AAC devices may help support AAC access for individuals with severe physical impairments across the lifespan, with motor (imagery)-based BCI-AAC switches showing feasibility as an input method for AAC control via scanning paradigms. Two participants with concern for cognitive impairment, but largely intact motor abilities demonstrated the greatest levels of BCI-AAC performance. Placed into a clinical context, that improved motor abilities may aid BCI-AAC outcomes supports consideration of timely intervention strategies for BCI-AAC assessment and intervention, similar to commercial AAC access. However, to integrate BCI-AAC into existing clinical frameworks such as feature matching, further research needs to be completed regarding the development of feature matching-based assessment tools for BCI-AAC (K. M. Pitt \& J. S. Brumberg, 2018b), and how an individual's profile (e.g., medications, food intake) impacts BCI-AAC learning.

Acknowledgments The authors would like to thank Chavis Lickvar-Armstrong and Dr. Jeremy Burnison for their assistance with this project.

Funding This work was supported in part by the Texas Woman's University Woodcock Institute Research Grant, the ASHA Foundation New Century Scholars Grant, the University of Kansas Summer Research Scholarship, and the National Institutes of Health (NIDCD R01- DC016343). 


\section{References}

Ahn, M., \& Jun, S. C. (2015). Performance variation in motor imagery brain-computer interface: A brief review. Journal of Neuroscience Methods, 243, 103-110. https:// doi.org/10.1016/i.jneumeth.2015.01.033

Bacci, E. D., Staniewska, D., Coyne, K. S., Boyer, S., White, L. A., Zach, N., \& Cedarbaum, J. M., \& Pooled Resource Open-Access ALS Clinical Trials Consortium. (2016). Item response theory analysis of the amyotrophic lateral sclerosis functional rating scale-revised in the pooled resource open-access ALS clinical trials database. Amyotrophic Lateral Sclerosis \& Frontotemporal Degeneration, 17(3-4), 157-167. https://doi.org/10.3109/21678421.2015.1095930

Beckung, E., \& Hagberg, G. (2002). Neuroimpairments, activity limitations, and participation restrictions in children with cerebral palsy. Developmental Medicine and Child Neurology, 44(5), 309-316. https://doi.org/10.1017/ $\underline{\mathrm{s} 0012162201002134}$

Beukelman, D., \& Mirenda, P. (2013). Augmentative and alternative communication: Supporting children and adults with complex communication needs (4th ed.). Paul H. Brookes Publishing Co..

Beukelman, D. R., Fager, S., Ball, L., \& Dietz, A. (2007). AAC for adults with acquired neurological conditions: A review. Augmentative and Alternative Communication, 23(3), 230-242. https://doi.org/10.1080/07434610701553668

Blain-Moraes, S., Schaff, R., Gruis, K. L., Huggins, J. E., \& Wren, P. A. (2012). Barriers to and mediators of brain-computer interface user acceptance: Focus group findings. Ergonomics, 55(5), 516-525. https://doi.org/10.1080/00140139.2012. $\underline{661082}$

Brumberg, J. S., Pitt, K. M., Mantie-Kozlowski, A., \& Burnison, J. C. (2018). BrainComputer interfaces for augmentative and alternative communication: A tutorial. American Journal of Speech-Language Pathology, 27(1), 1-12. https://doi. org/10.1044/2017 AJSLP-16-0244

Brumberg, J. S., Burnison, J. D., \& Pitt, K. M. (2016). Using motor imagery to control brain-computer interfaces for communication. In D. D. Schmorrow \& C. M. Fidopiastis (Eds.), Foundations of augmented cognition: Neuroergonomics and operational neuroscience (pp. 14-25). Springer.

Cedarbaum, J. M., \& Stambler, N. (1997). Performance of the amyotrophic lateral sclerosis functional rating scale (ALSFRS) in multicenter clinical trials. Journal of the Neurological Sciences, 152(S1), S1-S9. https://doi.org/10.1016/ s0022-510x(97)00237-2

Daly, I., Billinger, M., Laparra-Hernández, J., Aloise, F., García, M. L., Faller, J., Scherer, R., \& Müller-Putz, G. (2013). On the control of brain-computer interfaces by users with cerebral palsy. Clinical Neurophysiology, 124(9), 1787-1797. https://doi. org/10.1016/j.clinph.2013.02.118

Eliasson, A.-C., Krumlinde-Sundholm, L., Rösblad, B., Beckung, E., Arner, M., Öhrvall, A.-M., \& Rosenbaum, P. (2006). The Manual Ability Classification System (MACS) for children with cerebral palsy: Scale development and evidence of validity and reliability. Developmental Medicine and Child Neurology, 48(7), 549-554. https:// doi.org/10.1111/j.1469-8749.2006.tb01313.x 
Elvrum, A.-K. G., Andersen, G. L., Himmelmann, K., Beckung, E., Öhrvall, A.-M., Lydersen, S., \& Vik, T. (2016). Bimanual fine motor function (BFMF) classification in children with cerebral palsy: Aspects of construct and content validity. Physical \& Occupational Therapy in Pediatrics, 36(1), 1-16. https://doi.org/10.3109/0194 2638.20124.975314

Fried-Oken, M., Mooney, A., \& Peters, B. (2015). Supporting communication for patients with neurodegenerative disease. NeuroRehabilitation, 37(1), 69-87. https://doi.org/10.3233/NRE-151241

Friedrich, E. V., McFarland, D. J., Neuper, C., Vaughan, T. M., Brunner, P., \& Wolpaw, J. R. (2009). A scanning protocol for a sensorimotor rhythm-based brain-computer interface. Biological Psychology, 80(2), 169-175. https://doi.org/10.1016/j. biopsycho.2008.08.004

Geisler, M. W., \& Polich, J. (1990). P300 and time of day: Circadian rhythms, food intake, and body temperature. Biological Psychology, 31 (2), 117-136. https://doi. org/10.1016/0301-0511(90)90012-L

Geronimo, A., Simmons, Z., \& Schiff, S. J. (2016). Performance predictors of brain-computer interfaces in patients with amyotrophic lateral sclerosis. Journal of Neural Engineering, 13(2), 026002. Article 026002 https://doi. org/10.1088/1741-2560/13/2/026002

Gosmanova, K. A., Carmack, C. S., Goldberg, D., Fitzpatrick, K., Zoltan, B., Zeitlin, D. M., Wolpaw, J.R., Maehle, O.A., Borge, A., \& Vaughan, T. M. (2017). EEG-based braincomputer interface access To Tobii Dynavox Communicator 5. Rehabilitation Engineering and AssistiveTechnology Society of North America, 4. https://www. resna.org/sites/default/files/conference/2017/pdf versions/cac/Gosmnova.pdf

Halder, S., Agorastos, D., Veit, R., Hammer, E. M., Lee, S., Varkuti, B., Bogdan, M., Rosenstiel, W., Birbaumer, N., \& Kübler, A. (2011). Neural mechanisms of braincomputer interface control. Neuroimage, 55(4), 1779-1790. https://doi. org/10.1016/j.neuroimage.2011.01.021

Hammer, E. M., Halder, S., Blankertz, B., Sannelli, C., Dickhaus, T., Kleih, S., Müller, K.-R., \& Kübler, A. (2012). Psychological predictors of SMR-BCI-AAC performance. Biological Psychology, 89(1), 80-86. https://doi.org/10.1016/j. biopsycho.2011.09.006

Johnson, J. M., Inglebret, E., Jones, C., \& Ray, J. (2006). Perspectives of speech language pathologists regarding success versus abandonment of AAC. Augmentative and Alternative Communication, 22(2), 85-99. https://doi. org/10.1080/07434610500483588

Kasahara, T., Terasaki, K., Ogawa, Y., Ushiba, J., Aramaki, H., \& Masakado, Y. (2012). The correlation between motor impairments and event-related desynchronization during motor imagery in ALS patients. BMC Neuroscience, 13(1). Article 66, 1-10. https://doi.org/10.1186/1471-2202-13-66

Kleih, S. C., \& Kübler, A. (2015). Psychological factors influencing brain-computer interface (BCI-AAC) performance. In Proceedings of the 2015 IEEE International Conference on Systems, Man, and Cybernetics (SMC) (pp. 3192-3196). Kowloon, China. https://doi.org/10.1109/SMC. 2015.554 
Liberati, G., Pizzimenti, A., Simione, L., Riccio, A., Schettini, F., Inghilleri, M., Mattia, D., \& Cincotti, F. (2015). Developing brain-computer interfaces from a user-centered perspective: Assessing the needs of persons with amyotrophic lateral sclerosis, caregivers, and professionals. Applied Ergonomics, 50, 139-146. https://doi. org/10.1016/j.apergo.2015.03.012

Light, J., McNaughton, D., Beukelman, D., Fager, S. K., Fried-Oken, M., Jakobs, T., \& Jakobs, E. (2019). Challenges and opportunities in augmentative and alternative communication: Research and technology development to enhance communication and participation for individuals with complex communication needs. Augmentative and Alternative Communication, 35(1), 1-12. https://doi.org /10.1080/07434618.2018.1556732

Lotte, F., \& Guan, C. (2010). Regularizing common spatial patterns to improve BCIAAC designs: Unified theory and new algorithms. IEEE Transactions on Biomedical Engineering, 58(2), 355-362. https://doi.org/10.1109/TBME.2010.2082539

McCarthy, J., Light, J., Drager, K., McNaughton, D., Grodzicki, L., Jones, J., Panek, E., \& Parkin, E. (2006). Re-designing scanning to reduce learning demands: The performance of typically developing 2-year-olds. Augmentative and Alternative Communication, 22(4), 269-283. https://doi.org/10.1080/00498250600718621

McHugh, M. L. (2012). Interrater reliability: The kappa statistic. Biochemia Medica, 22(3), 276-282. https://doi.org/10.11613/BM.2012.031

Meador, K. J. (1998). Cognitive side effects of medications. Neurologic Clinics, 16(1), 141-155. https://doi.org/10.1016/S0733-8619(05)70371-6

Myrden, A., \& Chau, T. (2015). Effects of user mental state on EEG-BCI- AAC performance. Frontiers in Human Neuroscience, 9. Article 308, 1- 11. https://doi. org/10.3389/fnhum.2015.00308

Neuper, C., Scherer, R., Reiner, M., \& Pfurtscheller, G. (2005). Imagery of motor actions: Differential effects of kinesthetic and visual-motor mode of imagery in single-trial EEG. Cognitive Brain Research, 25(3), 668-677. https://doi. org/10.1016/j.cogbrainres.2005.08.014

Nijboer, F., Birbaumer, N., \& Kübler, A. (2010). The influence of psychological state and motivation on brain-computer interface performance in patients with amyotrophic lateral sclerosis - a longitudinal study. Frontiers in Neuroscience, 4, 1-13 https://doi.org/10.3389/fnins.2010.00055

Oostenveld, R., \& Praamstra, P. (2001). The five percent electrode system for highresolution EEG and ERP measurements. Clinical Neurophysiology, 112(4), 713719. https://doi.org/10.1016/S1388-2457(00)00527-7

Pfurtscheller, G., \& Da Silva, F. L. (1999). Event-related EEG/MEG synchronization and desynchronization: Basic principles. Clinical Neurophysiology, 110(11), 18421857. https://doi.org/10.1016/s1388-2457(99)00141-8

Pitt, K. M., \& Brumberg, J. S. (2018b). A screening protocol incorporating braincomputer interface feature matching considerations for augmentative and alternative communication. Assistive Technology, 32 (3), 161-172. https://doi.org /10.1080/10400435.2018.1512175 
Pitt, K. M., \& Brumberg, J. S. (2018a). Guidelines for feature matching assessment of brain-computer interfaces for augmentative and alternative communication. American Journal of Speech-Language Pathology, 27(3), 950-964. https://doi. org/10.1044/2018 AJSLP-17-0135

Pitt, K. M., Brumberg, J. S., Burnison, J. D., Mehta, J., \& Juhi, K. (2019). Behind the scenes of non-invasive brain-computer interfaces: A review of electroencephalography signals, how they are recorded, and why they matter. ASHA Perspectives, 4(6), 1622-1636. https://doi.org/10. 1044/2019_PERS-19-00059

Pitt, K. M., Brumberg, J. S., \& Pitt, A. R. (2019). Considering augmentative and alternative communication research for brain-computer interface practice. Assistive Technology Outcomes and Benefits, 13(Summer), 1-20. www.atia.org/ $\underline{\text { atob }}$

Randolph, A. B., Jackson, M. M., \& Karmakar, S. (2010). Individual characteristics and their effect on predicting mu rhythm modulation. International Journal of HumanComputer Interaction, 27(1), 24-37. https://doi.org/10.1080/10447318.2011.53 $\underline{5750}$

Rimbert, S., Gayraud, N., Bougrain, L., Clerc, M., \& Fleck, S. (2019). Can a subjective questionnaire be used as brain-computer interface performance predictor? Frontiers in Human Neuroscience, 12. Article 529, 1- 11. https://doi.org/10.3389/ fnhum.2018.00529

Scherer, R., Billinger, M., Wagner, J., Schwarz, A., Tassilo Hettich, D., Bolinger, E., Garcia, M. L., Navarro, J., \& Müller-Putz, G. (2015). Thought-based row-column scanning communication board for individuals with cerebral palsy. Annals of Physical and Rehabilitation Medicine, 58(1), 14-22. https://doi.org/10.1016/j. rehab.2014.11.005

Talukdar, U., Hazarika, S. M., \& Gan, J. Q. (2019). Motor imagery and mental fatigue: Inter-relationship and EEG based estimation. Journal of Computational Neuroscience, 46(1), 55-76. https://doi.org/10.1007/s10827-018-0701-0

Thompson, D. E., Gruis, K. L., \& Huggins, J. E. (2014). A plug-and-play brain-computer interface to operate commercial assistive technology. Disability and Rehabilitation. Assistive Technology, 9(2), 144-150. https://doi.org/10.3109/17483107.2013.78 $\underline{5036}$

Thompson, M. C. (2018). Critiquing the concept of BCI-AAC illiteracy. Science and Engineering Ethics, 25(4), 1217-1233. https://doi.org/10.1007/ s11948-018-0061-1

Velliste, M., Perel, S., Spalding, M. C., Whitford, A. S., \& Schwartz, A. B. (2008). Cortical control of a prosthetic arm for self-feeding. Nature, 453 (7198), 1098-1101. https://doi.org/10.1038/nature06996

Vuckovic, A., \& Osuagwu, B. A. (2013). Using a motor imagery questionnaire to estimate the performance of a brain-computer interface based on object oriented motor imagery. Clinical Neurophysiology, 124 (8), 1586-1595. https://doi. org/10.1016/j.clinph.2013.02.016 
Woolley, S. C. (2014). ALS Cognitive Behavioral Screen manual. The Forbes Norris MDA/ALS Research Center.Woolley, S. C., York, M. K., Moore, D. H., Strutt, A. M., Murphy, J., Schulz, P. E., \& Katz, J. S. (2010). Detecting frontotemporal dysfunction in ALS: Utility of the ALS Cognitive Behavioral Screen (ALS-CBS). Amyotrophic Lateral Sclerosis, 11(3), 303-311. https://doi.org/10.3109/17482961003727954

Zhang, J. Z., Jadavji, Z., Zewdie, E., \& Kirton, A. (2019). Evaluating if children can use simple brain computer interfaces. Frontiers in Human Neuroscience, 13. Article 24, 1-7. https://doi.org/10.3389/fnhum.2019.00024

Zickler, C., Riccio, A., Leotta, F., Hillian-Tress, S., Halder, S., Holz, E., Staiger- Sälzer, P., Hoogerwerf, E. J., Desideri, L., Mattia, D., \& Kübler, A. (2011). A brain-computer interface as input channel for a standard assistive technology software. Clinical EEG and Neuroscience, 42(4), 236-244. https://doi.org/10.1177/155005941104 $\underline{200409}$ 


\section{Supplementary Materials}

Table 1

Participant information for those with a diagnosis of ALS

\begin{tabular}{|c|c|c|c|c|c|c|}
\hline $\begin{array}{l}\text { Participant } \\
\text { Number }\end{array}$ & Diagnosis & $\begin{array}{l}\text { Time since } \\
\text { diagnosis } \\
\text { (years.months) }\end{array}$ & Sex & $\begin{array}{l}\text { Age } \\
\text { (years) }\end{array}$ & $\begin{array}{l}\text { Primary } \\
\text { communication } \\
\text { method }\end{array}$ & Medications \\
\hline A1 & Bulbar ALS & 0.7 & $\mathrm{~F}$ & 64 & Verbal & Diabetes \\
\hline $\mathrm{A} 2$ & Spinal ALS & 1.11 & $\mathrm{M}$ & 38 & Verbal & $\begin{array}{l}\text { Radicava infusions, } \\
\text { Riluzole from } 12 / 16 \\
12 / 18, \text { muscle } \\
\text { relaxants, as needed }\end{array}$ \\
\hline A3 & Spinal ALS & 10.4 & $\mathrm{~F}$ & 48 & $\begin{array}{l}\text { Verbal and eye- } \\
\text { gaze (as } \\
\text { needed) }\end{array}$ & $\begin{array}{l}\text { Muscle relaxants, as } \\
\text { needed }\end{array}$ \\
\hline A4 & Spinal ALS & 3.5 & $\mathrm{M}$ & 57 & Verbal & $\begin{array}{l}\text { Riluzole } 2 \mathrm{x} / \text { day, } \\
\text { muscle relaxants, as } \\
\text { needed }\end{array}$ \\
\hline
\end{tabular}




\section{Table 2}

Cognitive scores for participants with ALS. The ALS-CBS has a maximum score of 20, with a total score of $<17$ indicating a concern for cognitive impairment. The BCI-AAC screener has a maximum score of 24 with lower scores possibly indicting an increased concern for the presence of BCI-AAC related skills.

\begin{tabular}{llc}
\hline Participant & $\begin{array}{c}\text { ALS-CBS } \\
\text { (total/20) }\end{array}$ & BCI \\
& & $(/ 24)$ \\
\hline A1 & 15 & 22 \\
A2 & 19 & 22 \\
A3 & 19 & 24 \\
A4 & 14 & 24 \\
\hline
\end{tabular}




\section{Table 3}

Participant with ALS results for motor control and manual ability. The ALS-Functional Rating Scale (ALS-FRS) has a maximum score of 40, with higher scores indicate decreased motor impairment. In comparison, for the Manual ability classification systems (MACS) and Bimanual fine motor function classification system (BFMF) both have a high score of 5, with lower scores indicate decreased motor impairment. $R O M=$ range of motion

\begin{tabular}{|c|c|c|c|c|c|}
\hline Participant & ALS-FRS & MACS & BFMF & $\begin{array}{l}\text { BCI screener: upper } \\
\operatorname{limb}\end{array}$ & BCI screener: lower limb \\
\hline A1 & 33 & 1 & 1 & No impairment & No impairment \\
\hline \multirow[t]{5}{*}{ A 2} & 30 & 2 & 2 & Limited ROM. & Limited ROM and \\
\hline & & & & Decreased fine motor & weakness \\
\hline & & & & control and ability to & Ambulatory with \\
\hline & & & & grasp/grip & assistance \\
\hline & & & & & Limb spasticity \\
\hline \multirow[t]{3}{*}{ A3 } & 15 & 5 & 5 & Highly limited finger & Non-ambulatory \\
\hline & & & & movement and ability & Minimal movement of \\
\hline & & & & to grasp & legs and thighs \\
\hline \multirow[t]{4}{*}{ A4 } & 26 & 2 & 3 & Limited ROM. & Ambulatory without \\
\hline & & & & Decreased fine motor & assistance. Participant \\
\hline & & & & control and ability to & reported legs as 'still \\
\hline & & & & grasp/grip & strong' \\
\hline
\end{tabular}


Table 4

Neurotypical participants Cohen's Kappa values and 95\% confidence intervals

\begin{tabular}{lllll}
\hline Participant & Session & Cohen's Kappa & $\mathbf{9 5 \%}$ CI upper & 95\% CI lower \\
& Number & & bound & bound \\
\hline T1 & 1 & 0.3825 & 0.4994 & 0.2757 \\
& 2 & 0.4465 & 0.5661 & 0.3268 \\
\hline T2 & 3 & 0.406 & 0.5079 & 0.304 \\
\hline & 1 & 0.6751 & 0.7932 & 0.5571 \\
& 2 & 0.6574 & 0.7637 & 0.5511 \\
\hline T3 & 1 & 0.7339 & 0.8141 & 0.6537 \\
\hline & 2 & 0.0615 & 0.2008 & -0.0778 \\
& 3 & 0.5679 & 0.688 & 0.4478 \\
\hline
\end{tabular}

Note: Cohen's Kappa values and 95\% confidence intervals (CI) for each of the three BCI-AAC training sessions for neurotypical participants T1-T3. 


\section{Table 5}

Cohen's Kappa values and 95\% confidence intervals for participant A1

\begin{tabular}{llll}
\hline Session Number & Cohen's Kappa & $\mathbf{9 5 \%}$ CI upper bound & $\mathbf{9 5 \%}$ CI lower bound \\
\hline 1 & 0.020 & 0.122 & -0.082 \\
2 & 0.445 & 0.582 & 0.308 \\
3 & 0.423 & 0.552 & 0.293 \\
4 & 0.377 & 0.520 & 0.235 \\
5 & 0.289 & 0.407 & 0.171 \\
6 & 0.522 & 0.641 & 0.403 \\
7 & 0.544 & 0.654 & 0.431 \\
8 & 0.223 & 0.325 & 0.122 \\
9 & 0.195 & 0.286 & 0.104 \\
10 & 0.243 & 0.354 & 0.132 \\
11 & 0.285 & 0.397 & 0.174 \\
12 & 0.425 & 0.560 & 0.290 \\
\hline
\end{tabular}

Note: Cohen's Kappa values and 95\% confidence intervals (CI) for each BCI-AAC training session for participant A1. 
Table 6

Cohen's Kappa values and 95\% confidence intervals for participant A2

\begin{tabular}{llll}
\hline Session Number & Cohen's Kappa & $\mathbf{9 5 \%}$ CI upper bound & 95\% CI lower bound \\
\hline 1 & 0 & 0.438 & -0.438 \\
2 & 0.17 & 0.283 & 0.059 \\
3 & -0.051 & 0.069 & -0.171 \\
4 & 0.551 & 0.249 \\
5 & 0.340 & 0.346 & 0.096 \\
6 & 0.221 & 0.197 & -0.024 \\
7 & 0.087 & 0.241 & 0.026 \\
8 & 0.134 & 0.355 & 0.089 \\
9 & 0.222 & 0.173 & -0.080 \\
10 & 0.046 & 0.331 & 0.027 \\
11 & 0.179 & 0.249 & 0.003 \\
\hline 12 & 0.126 & 0.241 & 0.021 \\
\hline
\end{tabular}

Note: Cohen's Kappa values and 95\% confidence intervals (CI) for each BCI-AAC training session for participant A2. 
Table 7

Cohen's Kappa values and 95\% confidence intervals for participant A3

\begin{tabular}{llll}
\hline Session Number & Cohens Kappa & $\mathbf{9 5 \%}$ CI upper bound & 95\% CI lower bound \\
\hline 1 & -0.016 & 0.306 & -0.339 \\
2 & -0.172 & 0.203 & -0.546 \\
3 & -0.036 & 0.051 & -0.125 \\
4 & -0.146 & 0.005 & -0.297 \\
5 & 0.085 & 0.237 & -0.068 \\
6 & -0.092 & 0.194 & -0.378 \\
7 & 0.059 & 0.167 & -0.049 \\
8 & -0.018 & 0.173 & -0.21 \\
9 & 0.0289 & 0.1767 & -0.1188 \\
10 & 0.1079 & 0.2843 & -0.0685 \\
11 & -0.0446 & 0.2508 & -0.34 \\
12 & 0.1256 & 0.2643 & -0.013 \\
\hline
\end{tabular}

Note: Cohen's Kappa values and 95\% confidence intervals (CI) for each BCI-AAC training session for participant A3. 


\section{Table 8}

Cohen's Kappa values and 95\% confidence intervals for participant A4

\begin{tabular}{llll} 
Session Number & Cohen's Kappa & $\mathbf{9 5 \%}$ CI upper bound & $\mathbf{9 5 \%}$ CI lower bound \\
\hline 1 & 0 & 0.021 & -0.021 \\
2 & -0.051 & 0.051 & -0.152 \\
3 & -0.044 & 0.091 & -0.179 \\
4 & 0.158 & 0.333 & -0.017 \\
5 & 0.315 & -0.002 \\
6 & 0.156 & 0.356 & 0.151 \\
7 & 0.253 & 0.488 & 0.258 \\
8 & 0.373 & 0.409 & 0.146 \\
9 & 0.278 & 0.409 & 0.215 \\
10 & 0.312 & 0.511 & 0.302 \\
11 & 0.407 & 0.591 & 0.345 \\
12 & 0.468 & 0.213 & -0.057 \\
\hline & 0.078 & & \\
\hline
\end{tabular}

Note: Cohen's Kappa values and 95\% confidence intervals (CI) for each BCI-AAC training session for participant A4. 